\title{
Duality of the spin and density dynamics for two-dimensional electrons with a spin-orbit coupling
}

\author{
I. V. Tokatly 1,2 and E. Ya. Sherman ${ }^{2,3}$ \\ ${ }^{1}$ Nano-Bio Spectroscopy Group and ETSF Scientific Development Centre, Dpto. Física de Materiales, Universidad del País Vasco \\ UPV/EHU, E-20018 San Sebastian, Spain \\ ${ }^{2}$ IKERBASQUE, Basque Foundation for Science, 48011 Bilbao, Spain \\ ${ }^{3}$ Department of Physical Chemistry, Universidad del País Vasco UPV/EHU, 48080 Bilbao, Spain \\ (Received 7 June 2010; revised manuscript received 4 August 2010; published 12 October 2010)
}

\begin{abstract}
We study spin dynamics in a two-dimensional electron gas with a pure gauge non-Abelian spin-orbit field, for which systems with balanced Rashba and Dresselhaus spin-orbit couplings, and the (110)-axis grown GaAs quantum wells are typical examples. We demonstrate the duality of the spin evolution and the electron-density dynamics in a system without spin-orbit coupling, which considerably simplifies and deepens the analysis of spin-dependent processes. This duality opens a venue for the understanding of this class of systems, highly interesting for their applications in spintronics, through known properties of the systems without spin-orbit coupling.
\end{abstract}

DOI: $10.1103 /$ PhysRevB.82.161305

PACS number(s): 72.25.-b

The understanding of spin dynamics in a two-dimensional (2D) electron gas with spin-orbit (SO) interaction is highly important both for theoretical and applied spintronics, ${ }^{1-4}$ including the design of devices with controlled spin transport. In many physically interesting situations the SO coupling can be elegantly described as an effective non-Abelian vector potential. $^{5-17}$ There exists a class of systems, where the SO coupling corresponds to a pure gauge non-Abelian field. Therefore it can be gauged away and the behavior of a physical system should map to that of a system without SO coupling.

The coupled spin-charge dynamics is commonly described using the diffusion approximation, where the rate of the spin precession is much less than the momentum scattering rate. ${ }^{18,19}$ Currently, high-mobility $2 \mathrm{D}$ structures, where the time scale of momentum relaxation is longer than the spin rotation time, ${ }^{20,21}$ became available. Since here spins can make several turns between collisions with impurities, the conventional Dyakonov-Perel mechanism ${ }^{18}$ is not applicable, ${ }^{22}$ and another type of analysis is required. In the present Rapid Communication, we solve this problem for systems with a pure gauge SO coupling, including quantum effects due to the weak localization. We show for this general class of systems the existence of a duality of observables allowing the spin dynamics to be fully mapped to the density dynamics in a system without SO coupling. As a result, several regimes, including magnetic field dependence of the spin dynamics, can easily be explored using a single formula.

We represent the Hamiltonian of a $2 \mathrm{D}$ electron gas with SO coupling as follows ${ }^{16,17}$ (we use units with $\hbar=1$ ):

$$
H=\frac{1}{2 m} \int d^{2} \rho \Psi^{+}\left(i \partial_{i}+\mathcal{A}_{i}\right)^{2} \Psi+W\left[\Psi^{+}, \Psi\right],
$$

where $\Psi(\boldsymbol{\rho})$ is a spinor field operator, and the functional $W\left[\Psi^{+}, \Psi\right]$ contains all spin-independent contributions, including the external potential, electron-electron interactions, and, possibly, the electron-phonon coupling. Here $m$ is the electron effective mass and $\mathcal{A}_{i}$ are $2 \times 2$ matrix-valued components of a non-Abelian $\mathrm{SU}(2)$ gauge field describing the
SO coupling. A pure gauge $\mathcal{A}_{i}$ can be removed by a local $\mathrm{SU}(2)$ transformation. The general form of a pure gauge vector potential, $\mathcal{A}_{i}=m \alpha_{i}(\mathbf{h} \cdot \boldsymbol{\sigma})$, corresponds to the following SO Hamiltonian:

$$
H_{\mathrm{so}}=\alpha(\mathbf{h} \cdot \boldsymbol{\sigma})(\mathbf{k} \cdot \boldsymbol{\nu}),
$$

where $\boldsymbol{\sigma}$ is a vector of Pauli matrices, $\alpha$ is the SO coupling constant, $\mathbf{h}$ is a three-component unit vector for the SO field direction, $\boldsymbol{\nu}$ is a $2 \mathrm{D}$ unit vector in the $(x, y)$ plane, and $\alpha_{i}$ $=\alpha \nu_{i}$. The two practically important systems described by the Hamiltonian of Eq. (2) are: (i) the balanced RashbaDresselhaus system ${ }^{23,24}$ with $\mathbf{h}=( \pm 1, \pm 1,0) / \sqrt{2}$ and $\boldsymbol{\nu}$ $=( \pm 1, \pm 1) / \sqrt{2}$, and (ii) the (110)-axis grown GaAs quantum well, ${ }^{25-28}$ where $\mathbf{h}=(0,0,1), \boldsymbol{\nu}=(1,0)$ with the well axes chosen with respect to the crystal axes as $x\|[1 \overline{1} 0], y\|[001]$, and $z \|[110]$. Both systems are expected to demonstrate highly anisotropic spin-relaxation times with the spin component along the $\mathbf{h}$ axis having a very low relaxation rate, arising only due to a spin-dependent disorder. ${ }^{29}$ Spin currents in the thermodynamical equilibrium state, ${ }^{30}$ being common for $2 \mathrm{D}$ electron systems with SO coupling, are absent ${ }^{16}$ in the structures described by the Hamiltonian in Eq. (2).

A local $\mathrm{SU}(2)$ transformation, which gauges away the above type of SO coupling is $\widetilde{\Psi}(\boldsymbol{\rho})=\mathbf{U}_{\mathcal{A}} \Psi(\boldsymbol{\rho})$, where

$$
\mathbf{U}_{\mathcal{A}}=\exp [\operatorname{im} \alpha(\mathbf{h} \cdot \boldsymbol{\sigma})(\boldsymbol{\rho} \cdot \boldsymbol{\nu})]
$$

The transformation, Eq. (3), renders invariant all spinindependent quantities, such as the charge and current densities while the spin-density transforms covariantly,

$$
\widetilde{\mathbf{S}}=\frac{1}{2} \operatorname{tr}\left\{\boldsymbol{\sigma} \mathbf{U}_{\mathcal{A}}^{-1}(\mathbf{S} \cdot \boldsymbol{\sigma}) \mathbf{U}_{\mathcal{A}}\right\} .
$$

When the SO coupling is gauged away the dynamics of the transformed spin density $\widetilde{\mathbf{S}}(\boldsymbol{\rho}, t)$ reduces to the spin dynamics in the electron gas without SO interaction. Then, the physical spin density $\mathbf{S}(\boldsymbol{\rho}, t)$ is restored by 


$$
\mathbf{S}=\frac{1}{2} \operatorname{tr}\left\{\boldsymbol{\sigma} \mathbf{U}_{\mathcal{A}}(\widetilde{\mathbf{S}} \cdot \boldsymbol{\sigma}) \mathbf{U}_{\mathcal{A}}^{-1}\right\},
$$

to obtain measurable results. Here we follow this guideline and show that this approach allows to describe all regimes of spin dynamics on the same footing.

We consider a 2D electron gas with the Hamiltonian of Eq. (1) and, initially, a uniform spin density $\mathbf{S}$ produced, for example, by a static magnetic field $\mathbf{B}$. At $t=0$ the magnetic field is released and the spin relaxes due to a disorder potential and other interactions. To describe this process we first eliminate the SO by the gauge transformation of Eq. (3). Using Eq. (4) we find that the initial uniform physical spin density is mapped to the spin texture $\widetilde{\mathbf{S}}(\boldsymbol{\rho}, 0)=\widetilde{\mathbf{S}_{\|}}(\boldsymbol{\rho}, 0)$ $+\widetilde{\mathbf{S}}_{\perp}(\boldsymbol{\rho}, 0)$, where the term

$$
\widetilde{\mathbf{S}}_{\|}(\boldsymbol{\rho}, 0)=\mathbf{h}(\mathbf{S} \cdot \mathbf{h}),
$$

being parallel to $\mathbf{h}$, is untouched by the transformation and remains uniform, while the orthogonal to $\mathbf{h}$ part transforms into the helix structure ${ }^{31,32}$

$$
\widetilde{\mathbf{S}}_{\perp}(\boldsymbol{\rho}, 0)=[\mathbf{S}-\mathbf{h}(\mathbf{S} \cdot \mathbf{h})] \cos \left(\mathbf{Q}_{\mathrm{hx}} \cdot \boldsymbol{\rho}\right)-(\mathbf{S h}) \sin \left(\mathbf{Q}_{\mathrm{hx}} \cdot \boldsymbol{\rho}\right),
$$

where $\mathbf{Q}_{\mathrm{hx}}=2 m \alpha \boldsymbol{\nu}$ is the helix wave vector.

Since in the transformed system there is no SO coupling, the uniform part of the initial spin distribution, Eq. (6), is constant in time. A nontrivial dynamics occurs in the orthogonal channel due to a diffusional decay of the initial helix spin texture described by Eq. (7),

$$
\widetilde{S}_{\perp}^{\beta_{1}}(\boldsymbol{\rho}, t)=\int \mathcal{D}^{\beta_{1} \beta_{2}}\left(\boldsymbol{\rho}-\boldsymbol{\rho}^{\prime}, t\right) \widetilde{S}_{\perp}^{\beta_{2}}\left(\boldsymbol{\rho}^{\prime}, 0\right) d^{2} \boldsymbol{\rho}^{\prime},
$$

where $\mathcal{D}^{\beta_{1} \beta_{2}}\left(\boldsymbol{\rho}-\boldsymbol{\rho}^{\prime}, t\right)$ is the exact spin-diffusion Green's function of a $2 \mathrm{D}$ electron gas, which takes into account the disorder, electron-electron and electron-phonon interactions. To proceed further we note that in a nonmagnetic system without SO coupling the spin-diffusion Green's function is diagonal in the spin subspace $\mathcal{D}^{\beta_{1} \beta_{2}}(\boldsymbol{\rho}, t)=\delta_{\beta_{1} \beta_{2}} \mathcal{D}(\boldsymbol{\rho}, t)$. Hence Eq. (8) simplifies as

$$
\widetilde{\mathbf{S}}_{\perp}(\boldsymbol{\rho}, t)=\widetilde{\mathbf{S}}_{\perp}(\boldsymbol{\rho}, 0) \mathcal{D}\left(Q_{\mathrm{hx}}, t\right),
$$

where $\mathcal{D}(q, t)$ is a Fourier component of the spin-diffusion Green's function

$$
\mathcal{D}(q, t)=\int d^{2} \boldsymbol{\rho} e^{-i(\mathbf{q} \cdot \boldsymbol{\rho})} \mathcal{D}(\boldsymbol{\rho}, t)
$$

and we have taken into account that only the Fourier components of $\mathcal{D}(\boldsymbol{\rho}, t)$ with the modulus of the wave vector $q$ $=Q_{\mathrm{hx}}$ contribute to the dynamics of the helix in Eq. (7). Since the time-dependent factor in Eq. (9) is scalar, the transformation back to the physical spin, Eq. (5), simply reduces to removing tildas and the coordinate dependence in Eq. (9). Thus, we get the following exact result for the observable spin evolution:

$$
\mathbf{S}_{\perp}(t)=\mathbf{S}_{\perp}(0) \int \frac{d \omega}{2 \pi} \mathcal{D}\left(Q_{\mathrm{hx}}, \omega\right) e^{-i \omega t} .
$$

In Eq. (11) we represented $\mathcal{D}(q, t)$ via the Fourier integral because in the $\omega$ domain there is a simple expression of the spin-diffusion Green's function $\mathcal{D}(q, \omega)$ in terms of the spinspin correlator (the spin response function) $\chi_{\beta \beta}^{[S]}(q, \omega)$, where $\beta=(x, y, z)$ corresponds to the spin component

$$
\mathcal{D}\left(Q_{\mathrm{hx}}, \omega\right)=\frac{1}{i \omega}\left\{\frac{\chi_{\beta \beta}^{[S]}\left(Q_{\mathrm{hx}}, \omega\right)}{\chi_{\beta \beta}^{[S]}\left(Q_{\mathrm{hx}}, 0\right)}-1\right\} .
$$

This equation can be derived by considering a linear response on a time-dependent magnetic field that is adiabatically switched on at $t=-\infty$ and then suddenly switched off at $t=0$, i.e., $\mathbf{B}(t)=e^{\delta t} \theta(-t) \mathbf{B}$ with $\delta \rightarrow 0$ (see, e.g., Ref. 33 for similar calculations). Usually the SO coupling is weak on the Fermi energy scale, which implies $Q_{\mathrm{hx}} \ll k_{F}$, where $k_{F}$ is the Fermi momentum. Therefore in most situations one can safely replace the static $\omega=0$ response function in Eq. (12) by the macroscopic Pauli spin susceptibility, $\chi_{\mathrm{P}}$.

Equations (11) and (12) are the result of the spin-density dynamics duality and give the exact evolution of the uniform spin density. The problem is solved by mapping the spin relaxation in the physical system to the "washing out" an inhomogeneous spin texture in a dual system without SO coupling. The real spin relaxes because of SO-induced precession and randomness introduced by disorder, phonons, and interelectron interactions. ${ }^{34}$ For the transformed spin, it is the evolution of the spatially nonuniform spin-density distributions. An exact feature of the dynamics is that the vector $\mathbf{S}_{\perp}(t)$ always stays collinear to its initial direction. In the transformed picture (i.e., in the dual system) this is related to the diagonal structure of the spin response in a nonmagnetic electron gas. For the real system this translates to the fact that spins of electrons with opposite momenta process around the $\mathbf{h}$ axis in the opposite directions with the same rate.

At the level of the random-phase approximation the spin response function $\chi_{\beta \beta}^{[S]}(q, \omega)$ is equal to the density response function $\chi(q, \omega)$ of a noninteracting, but possibly disordered and/or coupled to phonons electron gas, while the Pauli susceptibility $\chi_{\mathrm{P}}$ is proportional to the compressibility $\partial n / \partial \mu$, with $n$ and $\mu$ being the electron concentration and chemical potential, respectively. Hence the spin-diffusion Green's function entering Eq. (11) reduces to

$$
\mathcal{D}\left(Q_{\mathrm{hx}}, \omega\right)=\frac{1}{i \omega}\left\{\frac{\chi\left(Q_{\mathrm{hx}}, \omega\right)}{\partial n / \partial \mu}-1\right\},
$$

which is exactly the density diffusion Green's function. Therefore in this physically important case the spin relaxation is mapped to the ordinary density diffusion.

Now we apply Eqs. (11) and (13) to a noninteracting disordered $2 \mathrm{D}$ electron gas with a momentum relaxation time $\tau$ and study possible regimes of spin dynamics. The densitydensity correlator $\chi\left(Q_{\mathrm{hx}}, \omega\right)$ can be obtained either diagrammatically or by solving the kinetic equation. In the semiclassical regime, corresponding to the summation of ladder diagrams, one obtains 


$$
\begin{gathered}
\mathcal{D}\left(Q_{\mathrm{hx}}, \omega\right)=\frac{\mathcal{K}\left(Q_{\mathrm{hx}}, \omega\right)}{1-\mathcal{K}\left(Q_{\mathrm{hx}}, \omega\right)}, \\
\mathcal{K}\left(Q_{\mathrm{hx}}, \omega\right)=\frac{1}{2 \pi} \int \frac{d \theta}{1-i \omega \tau+i \Omega_{\mathrm{so}} \tau \cos \theta},
\end{gathered}
$$

where the only SO-dependent parameter in the problem $\Omega_{\mathrm{so}} \equiv Q_{\mathrm{hx}} v_{F}$ ( $v_{F}$ is the Fermi velocity) is the maximum spin precession rate and $\Omega_{\mathrm{so}} \tau=\ell Q_{\mathrm{hx}}$ (electron mean free path $\ell$ $\left.=v_{F} \tau\right)$ characterizes the relaxation regime.

We begin with the pure diffusion $\Omega_{\text {so }} \tau \ll 1$ regime, , studied in the coordinate representation in Ref. 17. Here the Green's function, Eq. (14), reduces to

$$
\mathcal{D}\left(Q_{\mathrm{hx}}, \omega\right)=\frac{1}{D Q_{\mathrm{hx}}^{2}-i \omega},
$$

where $D=v_{F}^{2} \tau / 2$ is the diffusion coefficient. Inserting $\mathcal{D}\left(Q_{\mathrm{hx}}, \omega\right)$ of Eq. (16) into Eq. (11) we obtain

$$
\mathbf{S}_{\perp}(t)=\mathbf{S}_{\perp}(0) \exp \left(-D Q_{\mathrm{hx}}^{2} t\right),
$$

which exactly corresponds to the Dyakonov-Perel' mechanism with the spin-relaxation rate $\Gamma_{s}=D Q_{\mathrm{hx}}^{2}$. Moreover, the factor $1 / 2$ in the definition of $D$ acquires an interesting physical meaning in terms of the spin precession: it corresponds to the angular averaging of the precession rate $\left\langle\Omega_{\text {so }}^{2}(\mathbf{k})\right\rangle$ $=\Omega_{\mathrm{so}}^{2} / 2$.

The opposite, clean limit $\Omega_{\mathrm{so}} \tau \gg 1$, in terms of the dual (transformed) system corresponds to a reversible, purely ballistic washing out the helix texture. In this regime $\mathcal{D}\left(Q_{\mathrm{hx}}, \omega\right) \approx \mathcal{K}\left(Q_{\mathrm{hx}}, \omega\right)$ (see Ref. 35) and the integration in Eqs. (11) and (15) yields

$$
\mathbf{S}_{\perp}(t)=\mathbf{S}_{\perp}(0) J_{0}\left(\Omega_{\mathrm{so}} t\right)=\mathbf{S}_{\perp}(0) J_{0}\left(Q_{\mathrm{hx}} v_{F} t\right),
$$

where $J_{0}\left(\Omega_{\mathrm{so}} t\right)$ is the Bessel function. The same result can be derived directly from the microscopic spin precession about the $\mathbf{h}$ axes with a $\mathbf{k}$-dependent rate $\Omega_{\mathrm{so}}(\mathbf{k})=\Omega_{\mathrm{so}} \cos \phi$, where $\phi$ is the angle between $\mathbf{k}$ and $\boldsymbol{\nu}$. Indeed, the net result of the inhomogeneous precession reproduces Eq. (18),

$$
\mathbf{S}_{\perp}(t)=\mathbf{S}_{\perp}(0) \int \cos \left(\Omega_{\mathrm{so}} t \cos \phi\right) \frac{d \phi}{2 \pi}=\mathbf{S}_{\perp}(0) J_{0}\left(\Omega_{\mathrm{so}} t\right) .
$$

Note that the oscillations of the total spin generally occur in systems with SO coupling in the ballistic regime. However the decay of the amplitude of oscillations [Bessel function in Eq. (19)] is a feature of the pure gauge SO interaction. In the case of only Rashba or only Dresselhause coupling, the spins of all electrons at the Fermi contour rotate at the same rate. As a result the total spin demonstrates a purely oscillatory behavior with a constant amplitude. If both Rashba and Dresselhaus terms with unbalances strengths are present, the total spin oscillates with a decreasing but nonvanishing amplitude. Importantly, even weak disorder destroys the above qualitative difference, leading to a decay of oscillations for any SO coupling.

An intermediate regime of $\Omega_{\mathrm{so}} \tau \sim 1$, can be investigated numerically. The results are presented in Fig. 1. One can clearly see a crossover from the oscillating Bessel function like behavior to the exponential Dyakonov-Perel' decay. At

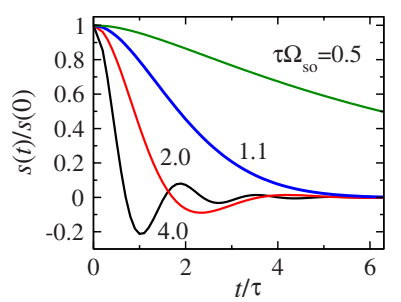

FIG. 1. (Color online) Time dependence of the spin for different parameters of SO coupling, shown near the plots, with $s(t)$ defined as $\mathbf{S}_{\perp}(t) \equiv s(t) \mathbf{S}_{\perp}(0) / S_{\perp}(0)$.

short times, the behavior of spin is universal: $\mathbf{S}_{\perp}(t)$ $=\mathbf{S}_{\perp}(0)\left(1-\Omega_{\mathrm{so}}^{2} t^{2} / 2\right)$ due to the unperturbed precession of the spins. For the density dynamics the universal short-time behavior is a direct consequence of the $f$-sum rule, ${ }^{36}$ as can be seen by expanding Eq. (11) at $t \rightarrow 0$.

Analysis of Eqs. (11), (14), and (15) shows that $\Omega_{\mathrm{so}} \tau=1$ is a critical point. With the decrease in $\Omega_{\mathrm{so}} \tau$ in the range $\Omega_{\mathrm{so}} \tau>1$, the first zero of $\mathbf{S}_{\perp}(t)$ rapidly shifts to larger times, with negative value regions becoming very shallow. At $\Omega_{\mathrm{so}} \tau<1$ zeroes of $\mathbf{S}_{\perp}(t)$ disappear and the dynamics is a pure decay.

The gauge transformation approach allows to analyze systems, where a direct treatment of the SO coupling would cause difficulties. The first effect we consider is the influence of the orbital motion in a nonquantizing magnetic field $B$ along the $z$ axis on the spin dynamics. We assume that due to a small $g$ factor of electron, the Zeeman coupling to the magnetic field does not cause a relevant spin precession. If $\Omega_{\text {so }} \tau \ll 1$, the density evolution at $B=0$ is diffusive and the electron mobility decreases with $B$ due to the Lorentz force as $\left(1+\omega_{c}^{2} \tau^{2}\right)^{-1}$, where $\omega_{c}=|e| B / m c$ is the cyclotron frequency. By the Einstein relation, the diffusion coefficient is renormalized by the same factor $D(B)=D(0) /\left(1+\omega_{c}^{2} \tau^{2}\right)$. Hence the spin-relaxation rate in Eq. (17) decreases as $\Gamma_{s}(B)=\Gamma_{s}(0) /\left(1+\omega_{c}^{2} \tau^{2}\right)$, which reproduces the results of the direct kinetic theory. ${ }^{37}$ For illustration, we consider the limit $\omega_{c} \tau \gg 1$ at short times $t \ll \tau$ in a more detail. Here the trajectories of electrons are nearly circles and the spin-independent kernel in Eq. (8) can be represented as (cf. Ref. 35)

$$
\mathcal{D}\left(\boldsymbol{\rho}^{\prime}-\boldsymbol{\rho}, t\right)=\frac{1}{2 \pi d(t)} \delta\left[\left|\boldsymbol{\rho}^{\prime}-\boldsymbol{\rho}\right|-d(t)\right]
$$

with the displacement $d(t)=2 R_{c}\left|\sin \left(\omega_{c} t / 2\right)\right|$, where $R_{c}$ $=v_{F} / \omega_{c}$ is the cyclotron radius and $R_{c} Q_{\mathrm{hx}}=\Omega_{\mathrm{so}} / \omega_{c}$. Straightforward integration in Eq. (8) yields [cf. Eq. (18)]

$$
\mathbf{S}_{\perp}(t)=\mathbf{S}_{\perp}(0) J_{0}\left[Q_{\mathrm{hx}} d(t)\right],
$$

where $Q_{\mathrm{hx}} d(t)=2 \Omega_{\mathrm{so}}\left|\sin \left(\omega_{c} t / 2\right)\right| / \omega_{c}$. For a very weak field (in a very clean system) $\omega_{c} \ll \Omega_{\text {so }}$, the result reproduces Eq. (18), as expected. In the opposite limit $\omega_{c} \gg \Omega_{\text {so }}$, no relaxation occurs. In terms of spin precession this can be understood $^{22,38,39}$ as very fast changes at the frequency $\omega_{c}$ in the direction of the SO field, keeping the total spin out of relaxation. In terms of the density dynamics, the electrons, forced to circulate around small radius cyclotron orbits, cannot spread out to destroy large scale $\sim 1 / Q_{\mathrm{hx}} \gg R_{c}$ density 
variations. At long times, the diffusion behavior takes over and the relaxation becomes exponential.

As a second example we briefly discuss the effect of weak localization on the spin relaxation ${ }^{40,41}$ by considering $\omega$-dependent renormalization of the momentum relaxation rate with the correction ${ }^{42-44}$

$$
\delta \tau_{\mathrm{wl}}^{-1}(\omega)=\frac{2}{\tau} \frac{1}{\partial n / \partial \mu} \int \frac{d^{2} q}{(2 \pi)^{2}} \frac{1}{D q^{2}-i \omega} .
$$

This correction arises due to the enhanced return probability, which slows down of the density dynamics and, when translated back to the physical system, eventually leads to the algebraic tail in spin relaxation at long time as $\mathbf{S}_{\perp}(t) \sim 1 / t$. $^{41}$ In terms of spin precession, the enhanced backscattering slows down the spin relaxation because upon the return to the initial point the electron spin direction remains the same.

To conclude, we have shown that in a wide class of systems the non-Abelian gauge field description of SO coupling reveals the duality of experimental observables and ensures the exact mapping of the spin dynamics to the density evolution. The evolution is described in terms of the response to an external perturbation with the wave vector equal to the spin helix wave vector $\mathbf{Q}_{\mathrm{hx}}$. We presented explicit results for a weak SO coupling with $Q_{\mathrm{hx}} \ll k_{F}$ valid for all systems of interest (this restriction is not required in general). This exact mapping opens a venue for understanding the whole class of practically important systems through better studied and more simple properties of the systems without SO coupling.

I.V.T. acknowledges funding by the Spanish MEC (Grant No. FIS2007-65702-C02-01), "Grupos Consolidados UPV/ EHU del Gobierno Vasco" (Grant No. IT-319-07) and the European Community through e-I3 ETSF project (Grant Agreement No. 211956). This work of E.Y.S. was supported by the University of Basque Country UPV/EHU under Grant No. GIU07/40, MCI of Spain under Grant No. FIS200912773-C02-01, and "Grupos Consolidados UPV/EHU del Gobierno Vasco” under Grant No. IT-472-10.
${ }^{1}$ R. Winkler, Spin-Orbit Coupling Effects in Two-Dimensional Electron and Hole Systems, Springer Tracts in Modern Physics (Springer, New York, 2003).

${ }^{2}$ I. Žutić et al., Rev. Mod. Phys. 76, 323 (2004); J. Fabian et al., Acta Phys. Slov. 57, 565 (2007).

${ }^{3}$ Spin Physics in Semiconductors, Springer Series in Solid-State Sciences, edited by M. I. Dyakonov (Springer, New York, 2008).

${ }^{4}$ M. W. Wu et al., Phys. Rep. 493, 61 (2010)..

${ }^{5}$ V. P. Mineev and G. E. Volovik, J. Low Temp. Phys. 89, 823 (1992).

${ }^{6}$ J. Fröhlich and U. M. Studer, Rev. Mod. Phys. 65, 733 (1993).

${ }^{7}$ I. L. Aleiner and V. I. Fal'ko, Phys. Rev. Lett. 87, 256801 (2001).

${ }^{8}$ L. S. Levitov and E. I. Rashba, Phys. Rev. B 67, 115324 (2003).

${ }^{9}$ Y. Lyanda-Geller, Phys. Rev. Lett. 80, 4273 (1998); Y. B. Lyanda-Geller and A. D. Mirlin, ibid. 72. 1894 (1994); J. B. Miller et al., ibid. 90, 076807 (2003).

${ }^{10}$ S.-R. Eric Yang and N. Y. Hwang, Phys. Rev. B 73, 125330 (2006).

${ }^{11}$ Q. Liu et al., Phys. Rev. B 76, 233409 (2007).

${ }^{12}$ N. Hatano et al., Phys. Rev. A 75, 032107 (2007).

${ }^{13}$ J.-S. Yang et al., Phys. Rev. B 78, 085312 (2008).

${ }^{14}$ B. W. A. Leurs et al., Ann. Phys. 323, 907 (2008).

${ }^{15}$ R. Raimondi and P. Schwab, EPL 87, 37008 (2009); M. Milletarì et al., ibid. 82, 67005 (2008).

${ }^{16}$ I. V. Tokatly, Phys. Rev. Lett. 101, 106601 (2008).

${ }^{17}$ I. V. Tokatly and E. Ya. Sherman, Ann. Phys. 325, 1104 (2010).

${ }^{18}$ M. I. Dyakonov and V. I. Perel', Sov. Phys. Solid State 13, 3023 (1972).

${ }^{19}$ P. Kleinert and V. V. Bryksin, Phys. Rev. B 79, 045317 (2009); T. D. Stanescu and V. Galitski, ibid. 75, 125307 (2007).

${ }^{20}$ M. Griesbeck et al., Phys. Rev. B 80, 241314 (2009).

${ }^{21}$ T. Korn, Phys. Rep. 494, 415 (2010). , and references therein.

${ }^{22}$ See, e.g., D. Culcer and R. Winkler, Phys. Rev. B 76, 195204 (2007).

${ }^{23}$ N. S. Averkiev and L. E. Golub, Phys. Rev. B 60, 15582 (1999).

${ }^{24}$ J. Schliemann et al., Phys. Rev. Lett. 90, 146801 (2003).
${ }^{25}$ M. I. Dyakonov and V. Yu. Kachorovskii, Fiz. Tekh. Poluprovodn. 20, 178 (1986) [Sov. Phys. Semicond. 20, 110 (1986)].

${ }^{26}$ G. M. Müller et al., Phys. Rev. Lett. 101, 206601 (2008).

${ }^{27}$ V. V. Bel'kov et al., Phys. Rev. Lett. 100, 176806 (2008).

${ }^{28}$ S. A. Tarasenko, Phys. Rev. B 80, 165317 (2009); Y. Zhou and M. Wu, EPL 89, 57001 (2010).

${ }^{29}$ M. M. Glazov and E. Ya. Sherman, Phys. Rev. B 71, 241312(R) (2005); V. K. Dugaev et al., ibid. 80, 081301 (2009); M. M. Glazov et al., ibid. 81, 115332 (2010); M. M. Glazov et al., Physica E (Amsterdam) 42, 2157 (2010).

${ }^{30}$ E. I. Rashba, Phys. Rev. B 68, 241315 (2003).

${ }^{31}$ B. A. Bernevig et al., Phys. Rev. Lett. 97, 236601 (2006).

${ }^{32}$ J. D. Koralek et al., Nature (London) 458, 610 (2009).

${ }^{33}$ D. Belitz and T. R. Kirkpatrick, Rev. Mod. Phys. 66, 261 (1994).

${ }^{34}$ M. M. Glazov and E. L. Ivchenko, Pis'ma Zh. Eksp. Teor. Fiz. 75, 476 (2002) [JETP Lett. 75, 403 (2002)]; M. M. Glazov and E. L. Ivchenko, Sov. Phys. JETP 99, 1279 (2004); D. Stich et al., Phys. Rev. Lett. 98, 176401 (2007).

${ }^{35}$ In the time-coordinate representation in this limit $\mathcal{D}\left(\boldsymbol{\rho}^{\prime}-\boldsymbol{\rho}, t\right)$ $=\delta\left(\left|\boldsymbol{\rho}^{\prime}-\boldsymbol{\rho}\right|-v_{F} t\right) /\left(2 \pi v_{F} t\right)$.

${ }^{36} \mathrm{G}$. Giuliani and G. Vignale, Quantum Theory of the Electron Liquid (Cambridge University Press, England, Cambridge, 2005).

${ }^{37}$ E. L. Ivchenko, Fiz. Tverd. Tela (Leningrad) 15, 1566 (1973) [Sov. Phys. Solid State 15, 1048 (1973)]; The Green's-function formalism, A. A. Burkov and L. Balents, Phys. Rev. B 69, 245312 (2004) in the presence of SO coupling leads to the same effect.

${ }^{38}$ M. M. Glazov, Solid State Commun. 142, 531 (2007).

${ }^{39}$ C. H. Chang et al., Phys. Rev. B 79, 125310 (2009).

${ }^{40}$ A. G. Mal'shukov et al., Phys. Rev. Lett. 76, 3794 (1996).

${ }^{41}$ I. S. Lyubinskiy and V. Yu. Kachorovskii, Phys. Rev. B 70, 205335 (2004).

${ }^{42}$ S. Hershfield and V. Ambegaokar, Phys. Rev. B 34, 2147 (1986).

${ }^{43}$ G. Strinati et al., Phys. Rev. B 40, 12237 (1989).

${ }^{44}$ V. K. Dugaev and D. E. Khmel'nitskii, Zh. Eksp. Teor. Fiz. Pis'ma Red. 90, 1871 (1986) [JETP Lett. 63, 1097 (1986)]. 